\title{
An Improved Multicast Routing Algorithm based on ADHOC Network
}

\author{
Yanhua Wang* and Yaqiu Liu
}

College of Information and Computer Engineering, Northeast Forestry University, Harbin, 150040, China

\begin{abstract}
After studying the topological structure of neighboring nodes in the WSN, this paper presents a local Combination Location (LCL) algorithm by combining principal manifold learning and the nonlinear dimension algorithm. This algorithm is particularly suitable for determining the relative locations of sensor nodes in large-scale, low-density WSNs, where the low connectivity between nodes and the large ranging error between long-distance nodes usually make accurate localization quite difficult. In this algorithm, based on the pair-wise distance between each node and its neighbour nodes within a certain communication range, the local geometry of the global structure is firstly obtained by constructing a local subspace for each node, and those subspaces are then aligned to give the internal global coordinates of all nodes. Combined with the global structure and the anchor node information, we can finally calculate the absolute coordinates of all unknown nodes by the least squares algorithm.
\end{abstract}

Keywords: wireless sensor network; node; localization algorithm

(Submitted on April 7, 2018; Revised on May 20, 2018; Accepted on June 29, 2018)

(C) 2018 Totem Publisher, Inc. All rights reserved.

\section{Introduction}

Node location in WSNs is finding the position relation between nodes based on the relationship between the distribution features and measurement information of all the nodes in the network, and then locating the coordinates of unknown nodes or determining the coordinates of nodes through the manifold of node distribution. In the field of machine learning, dimension reduction maps the data points from a high-dimensional space into a low-dimensional space, and it is intrinsically aimed at the distribution features of all the independent data points in a data set and their relative space information to find a mapping relation and realize the transformation from a high dimension to a low dimension. The features of transformed data points are maintained as much as possible, and their noise in high-dimensional space is eliminated. If the nodes in WSNs are regarded as independently distributed data points, and the measured information relation of adjacent nodes are seen as the relative space information of these data points, the node location in WSNs can be transformed into data dimension reduction in machine learning $[4,5]$. The technology of dimension reduction can reduce the redundant information of the original high-dimensional data and effectively find the essential structural features in data $[1,3,7]$. Therefore, the algorithms of node location in WSNs based on dimension reduction can tolerate certain noise in distance measurement, and some algorithms can even inhibit measurement noise, which makes them particularly suitable for node location when there is a large distance-measuring error $[8]$.

Many scholars have already started research in this area. Jenkins is a method of reducing Principal Component via the most classic analysis in machine learning (PCA) applied to the localization of WSNs, but because PCA is a linear dimensionality reduction method, in the case of large noise ranges, positioning accuracy will be relatively low. The location accuracy of a location algorithm is related to factors of many aspects, such as the number of anchor nodes, the communication capacity of a single sensor node, the area of the deployment zone, the density of sensor nodes and the distance-measuring error, etc. So, there have been a few commonly used location algorithms. In practical application, different location algorithms all have advantages and disadvantages. The location algorithm presented in this paper is mainly aimed at WSNs with a large and complex area of deployment and a low density of sensors. In a large and low-density area, generally, there would be distance-measuring errors between sensor nodes due to the influence of complex topography and anisotropy [6]. In addition,

\footnotetext{
* Corresponding author.

E-mail address: yanhuawangyhw@sina.com
} 
it may also cause cumulative distance-measuring errors because the communication distance is too long and the distance between nodes has to be measured through multi-hop communication [9].

In view of the large errors in location algorithms caused by distance measurement in a large and low-density area, based on the ideas of the principal manifold learning algorithm [10,11], this paper presents a local combination location algorithm. Usually, with the same method of distance measurement, the distance between two closer nodes can be measured more accurately. According to this criterion, adjacent nodes are first used to build a local subspace within a certain range of communication, and then all the local subspaces are combined to create a global coordinate system. Finally, through the global structure and the information of anchor nodes, the least square method is used to map out the absolute coordinates of all the nodes in the network.

\section{Local Combination Location Algorithm (LCL)}

A sensor node sample set $\left\{X_{1}, X_{2}, \ldots, X_{N}\right\}$ is given, in which $\mathrm{N}$ is the number of nodes, $x_{i}\left(x_{i} \in R^{d}\right)$ refers to the coordinates of the node $N=\{1,2, \ldots, N\}$, and $X_{1}$ is set to be at the origin of the coordinate system. Without loss of generality, the number of anchor nodes is represented by $\mathrm{M}(\mathrm{M}<<\mathrm{N})$. In order to obtain the neighboring node of a certain node, a network chart $\mathrm{G}$ is used to describe the topological structure of WSNs, in which each vertex represents a node in the network. If nodes $X_{i}$ and $X_{j}$ are within $\mathrm{CD}$, a certain range of communication, a side is supposed to exist between the vertexes $X_{i}$ and $X_{j}$ in the chart G. Assuming that the weight matrix of the chart $\mathrm{G}$ is $\mathrm{H}$, when there is a side between the vertexes $X_{i}$ and $X_{j}, H_{i j}=1$; or else, $H_{i j}=0$. So, the weight matrix is a symmetrical sparse matrix. It is shown in Equation (1).

$$
H_{i j}=\left\{\begin{array}{l}
1, \bar{d}_{i j}<C D \\
0, \bar{d}_{i j}>C D
\end{array} i, j=1 \ldots N\right.
$$

With regard to Line I of the weight matrix, if the number of elements the value of which is 1 is $\mathrm{k}$, it means the number of nodes adjacent to node $X_{i}$ is $\mathrm{k}$ within the communication range $\mathrm{R}$. The k adjacent nodes of the node $X_{i}$ is redefined as $\left\{X_{i 1}, X_{i 2}, \ldots, X_{i k}\right\}$, which also includes the node $X_{i}$ itself, so the distance matrix formed by the adjacent nodes of $X_{i}$ is $D^{(i)}=\left[\bar{d}_{p q}\right]$. Then, the coordinates of the inner product matrix can be obtained by Equation (2) by the following Equation $B^{(i)}=\left[b_{p q}\right]$ :

$$
b_{p q}=\left(\bar{d}_{1 p}^{2}+\bar{d}_{1 q}^{2}-\bar{d}_{p q}^{2}\right) / 2
$$

The inner product matrix here is a symmetric positive semi-definite matrix. In order to obtain the local coordinates, coordinate the inner product matrix eigenvalue decomposition. It is shown in Equation (3).

$$
B^{(i)}=V_{i} A_{i} V_{i}
$$

For each node, you can get all the $\Theta_{i}$ and then, based on all the $\Theta_{i}$ in the global space, the global coordinates of $T_{i}(i=1, \ldots, N)$ are constructed. It is shown in Equation (4).

$$
T_{i}=L_{i} \Theta_{i}+E_{i}
$$

In order to preserve the local position information in the global space as much as possible, we find the optimal affine 
transformation matrix $L_{i}$, which makes the $E_{i}$ minimum. It is shown in Equation (5).

$$
\sum_{i}\left\|E_{i}\right\|^{2}=\sum_{i}\left\|T_{i}-L_{i}\right\|^{2}=\min
$$

Obviously, for the determination of the global coordinate $T_{i}, E_{i}$ achieves the minimum optimal affine transformation matrix for $L_{i}=T_{i} \Theta_{i}^{+}$, the plug type available. It is shown in Equation (6).

$$
E_{i}=T_{i}\left(I-\Theta_{i}^{+}\right)
$$

After the local reconstruction error is obtained, the global reconstruction error can be solved. Let $T=\left[T_{1}, \ldots, T_{N}\right]$, then there is $T S_{i}=T_{i}$. The global reconstruction error is shown in Equation (7).

$$
\sum_{i}\left\|E_{i}\right\|^{2}=\sum_{i}\left\|T_{i}\left(I-\Theta_{i}^{+}\right)\right\|^{2}=\|T S W\|^{2}
$$

The eigenvalue decomposition of $\mathrm{R}$ is carried out, and $\mathrm{d}$ groups of eigenvectors corresponding to the $\mathrm{d}$ largest eigenvalues are selected as relative global coordinates in the d-dimensional space. After acquiring the global relative coordinates, in combination with the absolute coordinates of anchor nodes, the number of which is $\mathrm{n}$, all the global relative coordinates are transformed into global absolute coordinates through the transformation of coordinates. There is a problem of locating twodimensional node coordinates when there are at least 3 anchor nodes that are not on the same straight line. It is shown in Equation (8).

$$
x_{i}=C T_{i}+b
$$

$\mathrm{C}$ is the coordinate transformation matrix; B is the offset, which can be specified by the specific location of the anchor node. So, as long as the $\mathrm{C}$ can be obtained, the global relative coordinates to the global absolute coordinates can be realized. The arbitrary selection of an anchor node $X_{u}$ is given the coordinates of $x_{u}$. The process of solving the coordinate transformation matrix $\mathrm{C}$ is transformed to the following least square method. It is shown in Equation (9).

$$
\min _{c} \sum_{i=1}^{M}\left\|C \triangle T_{i}-\triangle x_{i}\right\|^{2}
$$

The absolute coordinates of the unknown nodes can be obtained by using the following Equation (10).

$$
\bar{x}_{i}=C\left(T_{i}-x_{u}\right)+x_{u}
$$

In this way, the whole LCL algorithm is completed, the procedures of which can be summarized as follows:

1. Extract local information. The weight matrix $\mathrm{H}$ of the network chart $\mathrm{G}$ is calculated and then aimed at each node $X_{i}$. The adjacent nodes are defined and the inner product matrix of coordinates $B^{i}$ is calculated. The eigenvalue decomposition of $B^{i}$ is carried out, and $W_{i}$ is calculated;

2. Restructure the global combined matrix. Iterative accumulation is used to calculate the global combined matrix R;

3. Calculate global relative coordinates. Eigenvalue decomposition is carried out on the global combined matrix, and d groups of eigenvectors corresponding to the d largest eigenvalues are selected as the global relative coordinates in the $\mathrm{d}$ - 
dimensional space;

4. Transformation of coordinates. In combination with enough number of anchor nodes, the least square method is used to transform relative global coordinates into absolute global coordinates.

In short, the process of LCL contains the following procedures: use a weight matrix to determine the number of nodes adjacent to a node and to generate local subspaces; combine all the local subspaces into a relative space of global coordinates containing all the nodes; then, in combination with enough anchor node coordinates, use the least square method to carry out the spatial transformation of coordinates to realize the location of coordinates of unknown nodes.

\section{Experiment Design and Discussion}

In order to verify the performance of the LCL algorithm, we need firstly test the proposed algorithm with an RSSI based measuring technique and then use the Noisy Disk model to simulate the performance of the LCL algorithm in cases of distance measuring error.

\subsection{Experimental Platform}

The distance measurement technique based on RSSI can get the distance from a signal point to the receiving point with a signal propagation attenuation model according to the strength or weakness of the received RSSI signal. Since RSSI is susceptible to the environment, there is no fixed propagation error model; instead, it needs to determine model parameters after an on-site survey as per the actual environment. Here, we chose CC2430 as the main chip to make experimental nodes. $\mathrm{CC} 2430$ is the first $2.4 \mathrm{GHz} \mathrm{RF}$ system single chip that complies with ZigBee technology [2]. It still uses the infrastructure of CC2420 and integrates the ZigBee RF front end, memory and micro-controller into a single chip. The current loss during its working is $27 \mathrm{~mA}$, which is respectively lower than $27 \mathrm{~mA}$ or $25 \mathrm{~mA}$ in the receiving or transmitting pattern. The hardware supports CSMA/CA function with digitized RSSI/LQI and strong DMA function. Each node can receive/send RF signal. When one node sends a signal, the other nodes can receive it and survey its RSSI. Define $P_{i j}$ as the strength $(m W$ ) of signal that is sent by node $\mathrm{j}$ and received by node $i$, and assume $P_{i j}=P_{j i} ; P_{i j}$ is a random variable of logarithmic normal distribution; $P_{i j}(\mathrm{dBm})=10 \log _{10} P_{i j}$ complies with Gaussian distribution. It is shown in Equation (11) and Equation (12).

$$
\begin{gathered}
P_{i j}(d B m) \sim N\left(P_{i j}(d B m), \sigma_{d B}^{2}\right) \\
\bar{P}_{i j}(d B m)=P_{0}(d B m)-10 n_{p} \log _{10}\left(d_{i j} / d_{0}\right)
\end{gathered}
$$

We used CC2430 to make nodes send and receive RF signals and measured the RSSI value to confirm the statistics model mentioned above. In order to get the above model, it requires determining three parameters: $d_{0}, P_{0}(d B m)$ and $n_{p}$. Usually $d_{0}=1 \mathrm{~m} ; P_{0}(\mathrm{dBm})$ is the measured RSSI value when the distance $d_{i j}=1 \mathrm{~m}$ is between node $X_{1}$ and $X_{2}$. When $d_{0}$ and $P_{0}(\mathrm{dBm})$ are both determined, put node $X_{1}$ and $X_{2}$ in different places; then, try several times to get the RSSI values of them. The mean value of $n_{p}$ is solved. Until this moment, three parameters of the RSSI signal transmit attenuation model are all obtained. The red circle in Figure1 is the average value of the RSSI signal measured at different distances from the sensor nodes. In this experiment, each position measures 10 RSSI values as well as the average. According to the measured value, the parameters of the RSSI signal transmission attenuation model can be $P_{0}(d B m) \approx-45, n_{p} \approx 3$. It is shown in Figure 1.

The experiment finds that for two fixed sensor nodes far away at certain distances, not only does the RSSI fluctuate, but with increasing distance, the vibration frequency and amplitude become bigger. Figure 2 gives the distribution of signal strength along with the time when two nodes of the transceiver stand away at distances of 1, 2, 3 meters; at each position, 105 groups of signals are observed; each group of the signal are at intervals of $2 \mathrm{~s}$. As seen, the RSSI value undulates around the mean value at a fixed position. The longer the distance is, the fiercer the fluctuation becomes. As shown in Figure 1 and Figure 2 , the above-mentioned model is in line with the actual situation. 


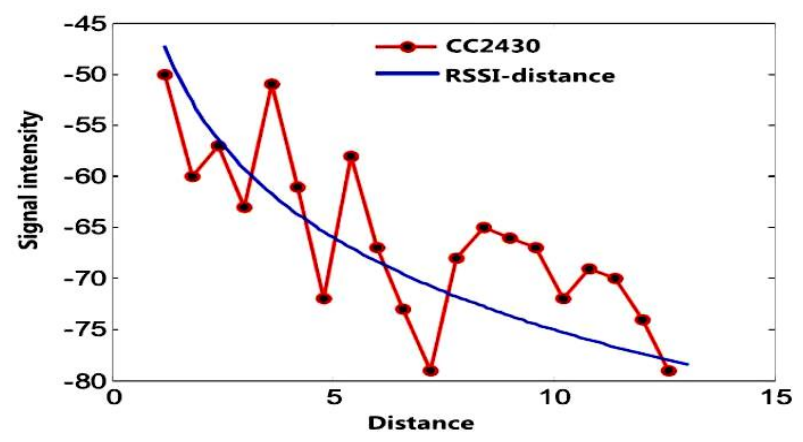

Figure 1. Relationship between RSSI and distance
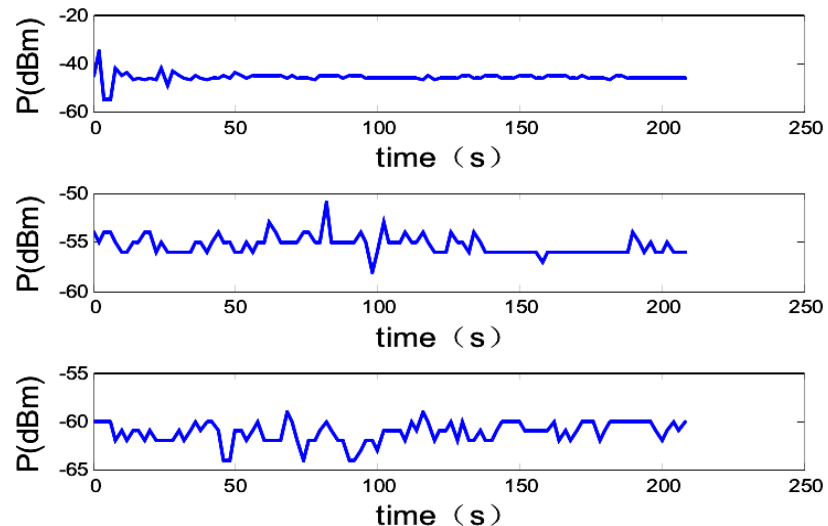

Figure 2. RSSI fluctuations with time at the distance of 1, 2, $3 \mathrm{~m}$ between two nodes

\subsection{Performance Evaluation}

A simulation is used to analyze and evaluate the performance of the LCL location algorithm. Matlab is chosen as the simulation platform, and all the algorithms are in the Matlab language. In all the simulation experiments, the sensor nodes are all deployed in a zone covering an area of $100 \times 100 \mathrm{~m} 2$. The performance of algorithms is evaluated with three factors, namely the communication distance of sensor nodes (CD), the number of sensor nodes, and the distance-measuring error of nodes $(\sigma)$. In Figure 3, the sensor nodes are deployed regularly and randomly, respectively, in which the red hollow diamonds are the anchor nodes with known coordinates and the blue circles are unknown nodes.

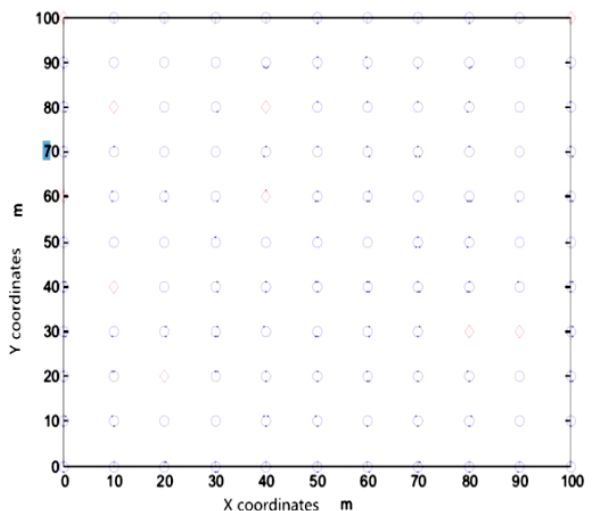

(a) Regularly deployed

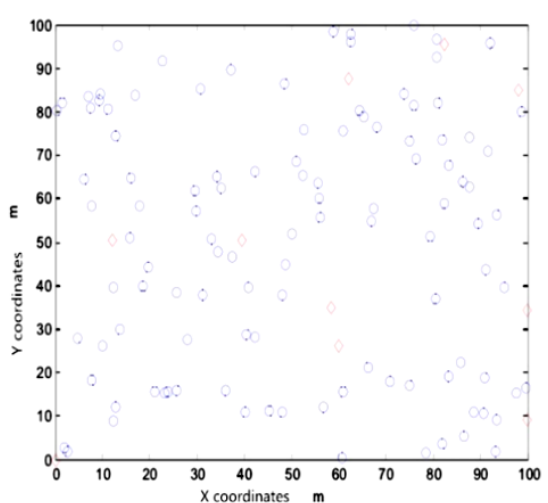

(b) randomly deployed

Figure 3. Regularly and randomly deployed sensor nodes

Simulation process, the measurement distance between nodes using the Disk Noisy model. It is shown in Equation (13).

$$
\bar{d}\left(x_{i}, x_{j}\right)=\left\{\begin{array}{l}
N\left(d\left(x_{i}, x_{j}\right), \sigma\right) \\
0
\end{array}\right.
$$


Generally, the communication capacity of sensor nodes is relatively stable, that is, the communication distance CD of sensor nodes is fixed. However, the range of WSNs deployment will vary with different practical applications. Of course, the overall network coverage can be achieved by increasing the number of nodes deployed, but it will undoubtedly increase the cost of network deployment. Figure 2 provides a chart of node deployment and a chart of network connection obtained when the communication distance of nodes CD is $40 \mathrm{~m}$ and the 100 sensor nodes (including 10 anchor nodes) are randomly deployed in the areas of $100 \times 100 \mathrm{~m}^{2}$ and $150 \times 150 \mathrm{~m}^{2}, 200 \times 200 \mathrm{~m}^{2}, 250 \times 250 \mathrm{~m}^{2}$ respectively.
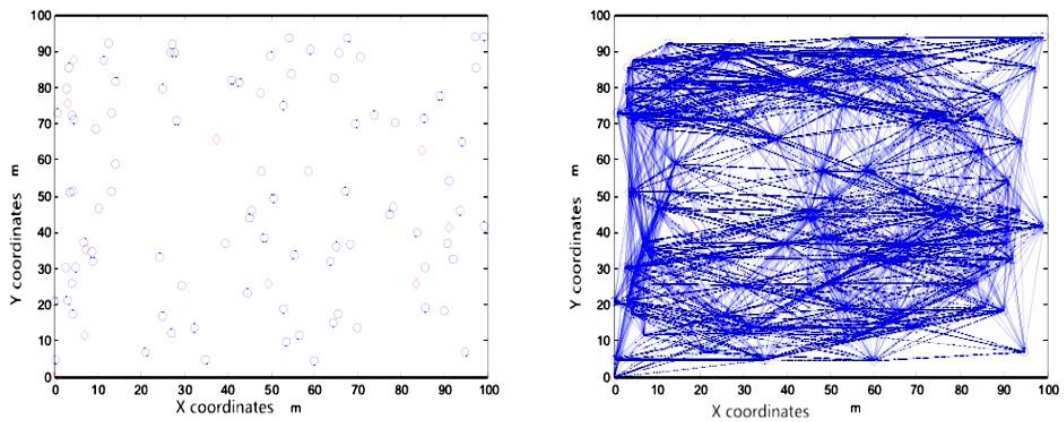

(a) Deployment area of $100 \times 100 \mathrm{~m}^{2}$ random deployment diagram
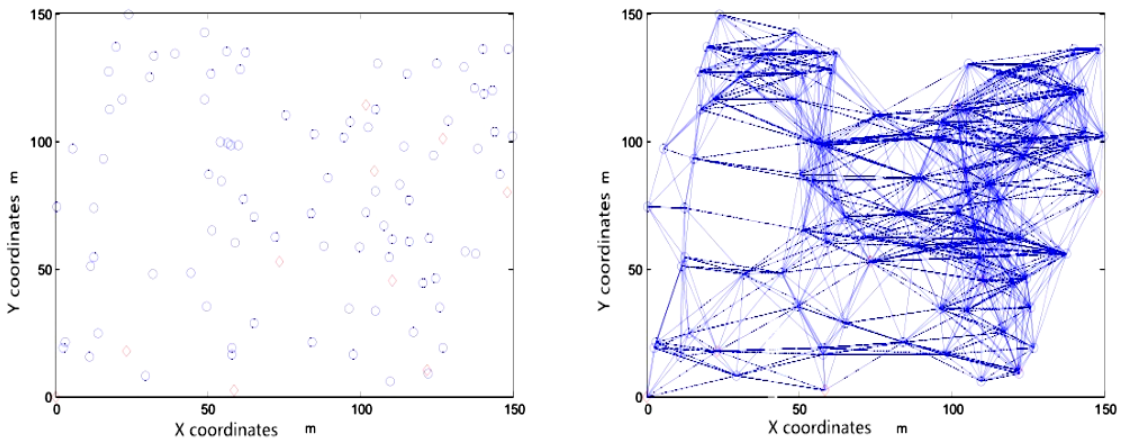

(b) Deployment area of $150 \times 150 \mathrm{~m}^{2}$ random deployment diagram
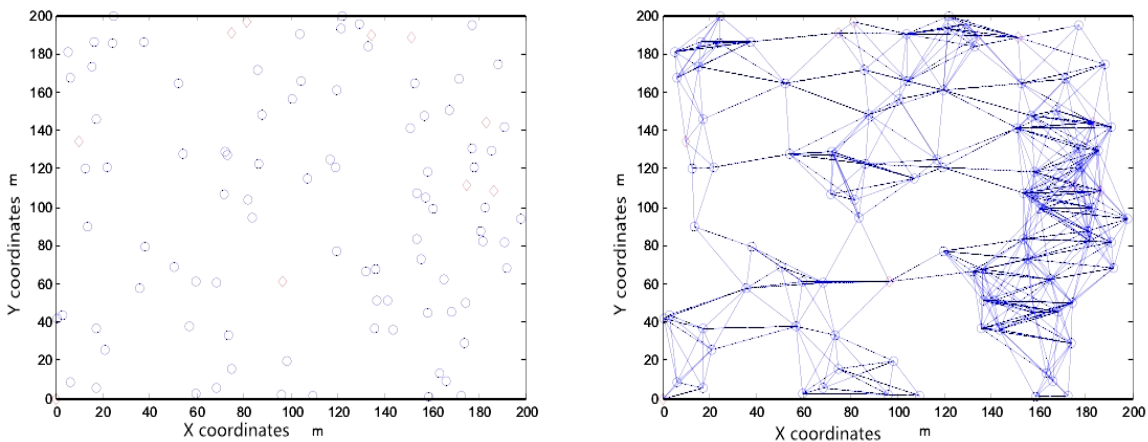

(c) Deployment area of $200 \times 200 \mathrm{~m}^{2}$ random deployment diagram
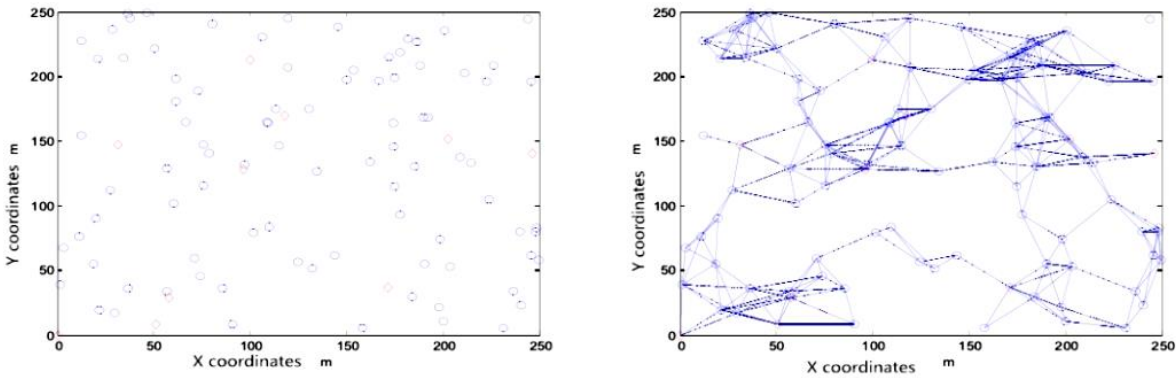

(d) Deployment area of $250 \times 250 \mathrm{~m}^{2}$ random deployment diagram Figure 4. Randomly deployed sensor nodes and network connectivity graphs $(\mathrm{NN}=100, \mathrm{CD}=40 \mathrm{~m})$ 
With the continuous expansion of the deployment range, more loopholes begin to emerge in the area covered by the network. As the CD is fixed, the connectivity of nodes will decrease. Within the zone covering an area of $250 \times 250 \mathrm{~m}^{2}$, isolated nodes have also appeared on the upper right corner (nodes that cannot communicate with other nodes in the network). Under this circumstance, the location of nodes will be directly influenced. Figure 4 provides the average connectivity of the network and the average number of adjacent anchor nodes in the network.

The average connectivity of the network is $30.62,18.46,9.5$ and 6.26 respectively. The average number of anchor nodes in the network is $2.9,1.79,0.87$ and 0.58 , respectively. From the above data, it can be seen that with the expansion of the deployment area, not only is the access speed of the network reduced, but also the average number of adjacent anchor nodes in the network is cut down. This will directly affect the location performance of some location algorithms that rely on the number of anchor nodes (such as the trilateral location algorithm). The communication distance of sensor nodes is related to hardware cost and directly affects the energy consumption of nodes. Strong communication capacity means it needs a higher consumption of energy. WSNs may be deployed in places with a vast area of land but few people. Sensor nodes are used only once. Therefore, reducing energy consumption and extending the service life of nodes has been major problems. As for the self-positioning technology of sensor nodes in the network, it is of great importance to study how highly accurate locations can be realized when the communication capacity of nodes is limited. When the deployment area of the sensor network is fixed, on the premise of not changing the number of nodes deployed, changes in the node communication distance will have a direct influence on the average access speed of the network.

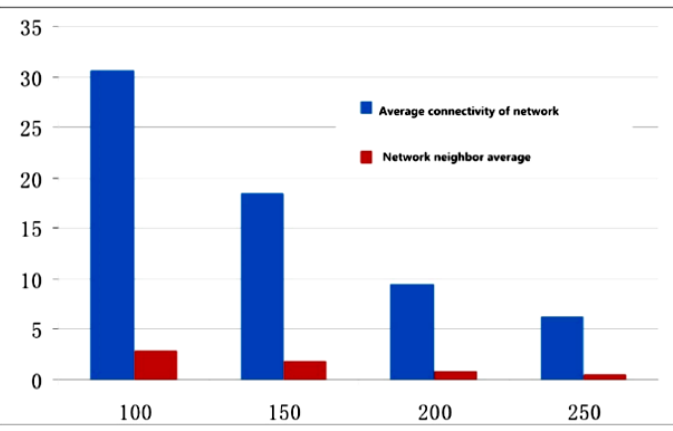

Figure 5. Average network connectivity and number of neighbor anchor nodes. (Deployment areas are $100 \times 100 \mathrm{~m}^{2}, 150 \times 150 \mathrm{~m}^{2}, 200 \times 200 \mathrm{~m}^{2}$, $250 \times 250 \mathrm{~m}^{2}$, respectively, $\mathrm{NN}=100$, anchor node number is $10, \mathrm{CD}=40 \mathrm{~m}$ )

Figure 5 provides a random deployment diagram of 100 sensor nodes in an area of $100 \times 100 \mathrm{~m}^{2}$ as well as a diagram of network node connection when CD changes in the range of $10 \mathrm{~m}-35 \mathrm{~m}$. It can be seen that as CD increases, the connectivity of nodes is continuously reinforced. Figure 6 provides bar graphs on the average network connectivity and the average the number of neighboring anchor nodes in the network. The average connectivity of the network is $3.1,5.72,9.68,14.4,19.42$ and 24.68, respectively. The average number of anchor nodes in the network is $0.32,0.59,1,1.45,1.85$ and 2.29 respectively.

Here, the number of sensor nodes (NN), the communication distance of sensor nodes (CD) and distance-measuring errors $\sigma$ are used as adjustable parameters to investigate their influences on the performance of the LCL algorithm. In the following three groups of simulation experiments, 100 nodes are randomly deployed in an area of $100 \times 100 \mathrm{~m}^{2}$, and 6 of them are selected randomly as anchor nodes with their position information already known.

First, quantitative analysis is made to analyze the performance of the LCL algorithm based on the number of nodes deployed. In a certain space for deployment, the number of sensor nodes is directly related to the hardware cost of the network, the routing algorithm of nodes, energy consumption of the network and network coverage. It is of great significance to choose a proper number of nodes, finish the needed monitoring of events and environmental parameters, and acquire the position information of sensor nodes. The distance-measuring effort $\sigma$ is set to $10 \%$, and the number of sensor nodes is increased from 20 to 100 when the node communication distance CD is $20 \mathrm{~m}, 30 \mathrm{~m}$ and $40 \mathrm{~m}$ respectively. The location error obtained is shown in Figure 7(a).

The $\mathrm{CD}$ is set to $40 \mathrm{~m}$, and the number of sensor nodes is increased from 20 to 100 when $\sigma$ is $10 \%, 20 \%$ and $30 \%$, respectively, with the location error shown in Figure 7(b). It can be seen that whether CD or A is fixed, increasing the number of sensor nodes will effectively improve the location accuracy. When the number of nodes is larger than 60, the results of the three kinds of $\mathrm{CD}$ have not shown relatively significant changes; in other words, if $\sigma$ and $\mathrm{CD}$ are both fixed, it can meet certain requirements of location error when the number of nodes reaches 60 . In this case, if an excessive number of nodes are deployed, it will not only waste resources but also increase the volume of network communication and shorten the service life 
of the network. $\sigma$ has a great influence on the LCL algorithm.

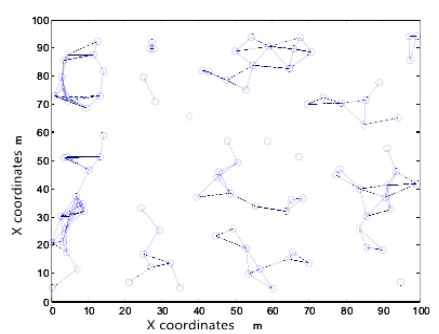

(a) $C D=10 \mathrm{~m}$

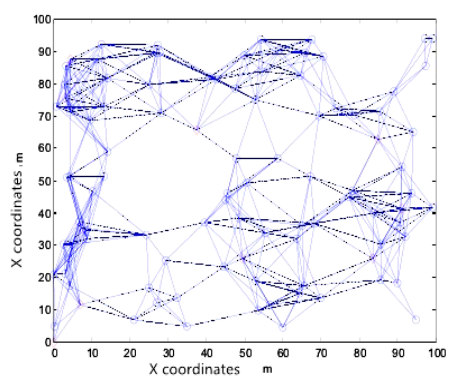

(c) $C D=20 \mathrm{~m}$

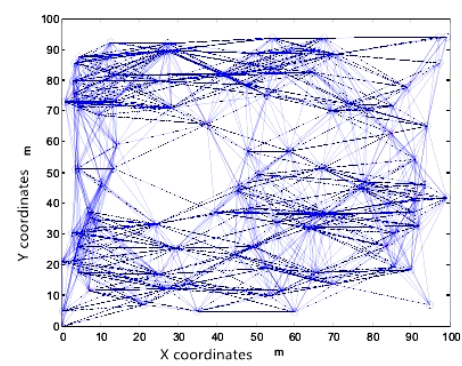

(e) $C D=30 \mathrm{~m}$

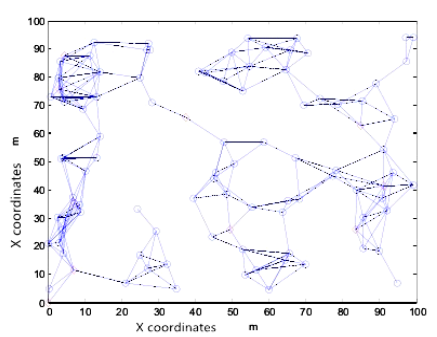

(b) $C D=15 \mathrm{~m}$

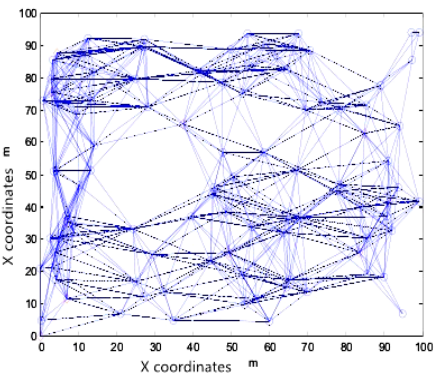

(d) $C D=25 \mathrm{~m}$

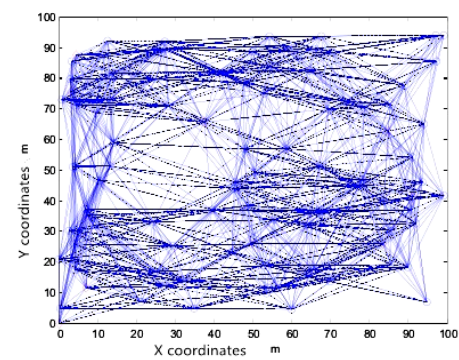

(f) $C D=35 \mathrm{~m}$

Figure 6. Network connectivity graphs when CD changes from $10 \mathrm{~m}$ to $30 \mathrm{~m}$

Take the node number 60 as an example, the positioning error of $\sigma=20 \%$ is increased by about $200 \%$ when the positioning error is more than $\sigma=10 \%$, and the positioning error of $\sigma=30 \%$ is about $450 \%$ when the position error is more than $\sigma=10 \%$. Moreover, only $\sigma=10 \%$. The number of nodes in the number of more than 40 of the positioning error will be relatively stable. The LCL algorithm is easily influenced as it mainly relies on the local subspaces composed of nodes adjacent to a node within a one-hop communication range. Therefore, if $\sigma$ within a one-hop range is very large, it will inevitably seriously affect the location error. Reducing the number of nodes from 100 to 20 has no significant influence on the average location error of the whole network, especially when the number of nodes is between 60 and 100 . Within a certain range of deployment space, as long as $\mathrm{A}$ is maintained at about $10 \%$, fewer nodes can also keep the location accuracy within a certain range, that is, the LCL algorithm satisfies the requirements of low-density WSNs locations, as shown in Figure 7(b).

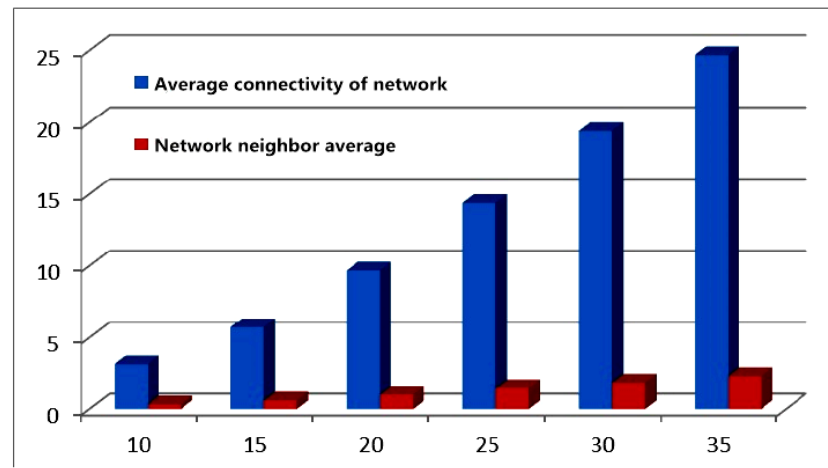

Figure 7. Average network connectivity and number of neighbor anchor nodes (Deployment area is $100 \times 100 m^{2}, \mathrm{NN}=100$, anchor node number is 10 ) 


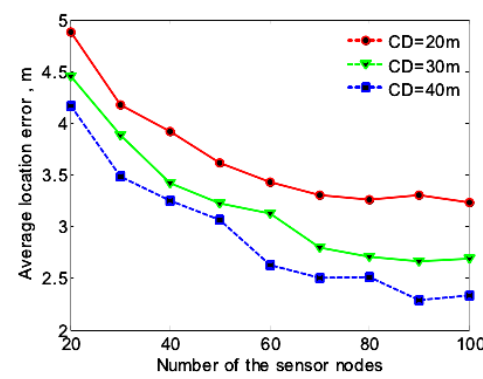

(a)

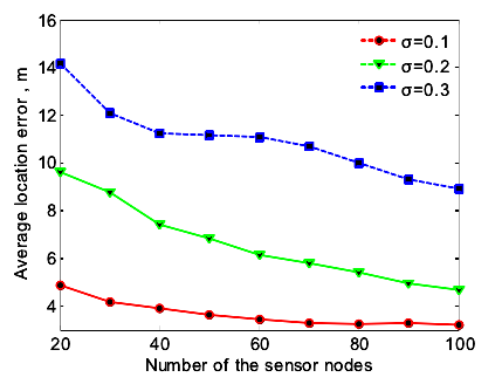

(b)

Figure 8. Average location error of LCL localization algorithm as a function of the sensor node number at $\sigma=10 \%$ (a) and $\mathrm{CD}=40 \mathrm{~m}$ (b)

Besides, the analysis is made on the influence of $\sigma$ on the LCL location algorithm. With different distance measurement technologies, $\sigma$ also varies. The experiment above has shown that $\sigma$ has a great influence on the location accuracy of the LCL location algorithm. Next, quantitative analysis will be made to determine the degree of influence. Set the communication distance $\mathrm{CD}=40 \mathrm{~m}$ for all the sensor nodes, the number of anchor nodes to 6 , the total number of sensor nodes in $\mathrm{NN}=100$, $\mathrm{NN}=70, \mathrm{NN}=30$, change from $5 \% \sigma$ ranging error between nodes within a jump to $40 \%$, and the positioning error results as shown in Figure $8(\mathrm{a})$. Set $\mathrm{NN}=100$, the number of anchor nodes is 6 , in the case of $\mathrm{CD}=20, \mathrm{CD}=30 \mathrm{~m}, \mathrm{CD}=40 \mathrm{~m}$, so that $\sigma$ is increased from $5 \%$ to $40 \%$. The resulting positioning error results are shown in Figure 8(b).

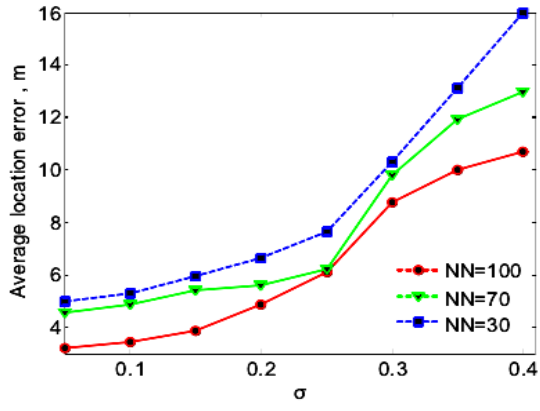

(a)

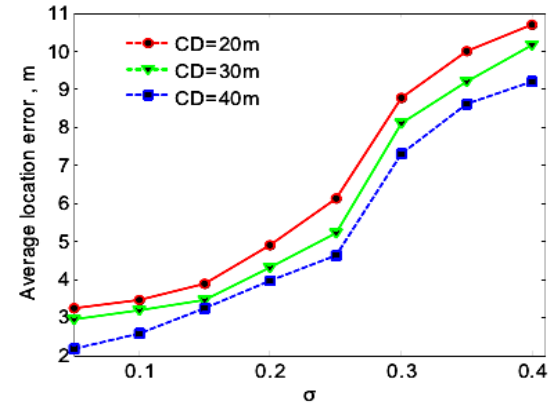

(b)

Figure 9. Average location error of LCL localization algorithm as a function of the sensor node ranging error at $\mathrm{CD}=40 \mathrm{~m}$ (a) and $\mathrm{NN}=100$ (b)

Lastly, the relationship between the CD and the LCL location algorithm is analyzed. From the experiment above, it can be preliminarily determined that $\mathrm{CD}$ has a steady influence on LCL. Next, a quantitative analysis is made of the relationship between them. CD not only is related to the hardware cost of nodes but also directly determines the battery consumption of sensor nodes and influences its service life. It is shown in Figure 9.

In conclusion, the performance of the LCL algorithm has been evaluated quantitatively in terms of the number of sensor nodes, the communication distance of nodes and distance-measuring errors. It can be seen that when the distance-measuring error between nodes is $10 \%$, the LCL algorithm can still maintain high location accuracy even though there are relatively a small number of sensor nodes. The distance-measuring error has a great influence on the location accuracy of the LCL algorithm. When the distance-measuring error is larger than $25 \%$ in particular, the location accuracy of the LCL algorithm slides down dramatically. The communication distance between nodes has a relatively stable influence on the LCL algorithm because as the communication capacity of nodes is increased, the location accuracy is also stably improved. As the LCL algorithm forms subspaces by using the adjacent nodes within one hop of sensor nodes and starts locating after combining all the subspaces to form the whole space covering the whole network, the distance-measuring error within one hop has a greater influence on the LCL algorithm. In general, higher distance-measuring accuracy can be achieved when nodes are closer, so the distance-measuring error is relatively small within one hop. To sum up, the LCL algorithm is suitable for the selfpositioning of WSNs nodes in a large area of low density.

\section{Conclusions}

This paper focuses on WSNs in large-scale and low-density environments, node distribution dispersion, limited communication ability and other practical problems. Through the study of the topological structure of neighboring nodes and the mainstream form of the learning algorithm, this paper presents a local Combination Location (LCL) algorithm. The 
algorithm is based on the distance between nodes, each node and its neighbor nodes of a local subspace; then, the local subspace combines into a global coordinate system and estimates the global relative coordinates of all nodes.

\section{Acknowledgements}

This work is supported by the Fundamental Research Funds for Central Universities (2572015BB24) and is supported by the National Natural Science Foundation of China (31370565).

\section{References}

1. J. Bouttier, P.Francesco, E. Guitter, “Geodesic Distance in Planar Graphs”, Nuclear Physics, Section B, vol.663, no.3, pp.535567,2013

2. P. Baronti, P. Pillai, "Wireless Sensor Networks: A Survey on the State of The Art and The 802.15. 4 and ZigBee Standards", Computer Communications, vol.30, no.7, pp. 1655-1695, 2007

3. H. Dai, A. Chen, X. Gu, "Localisation Algorithm for Large-scale and Low-density Wireless Sensor Networks". IET Electronics Letters, vol.47, no.15, pp.881-883, 2011

4. J. Juang, "Solving The Heterogeneous Positioning Problem Via Eigen-decomposition", IET Electronics Letters, vol.44, no.6, pp. 432-433, 2008

5. O. Jenkins, "Localization from Pairwise Distance Relationships Using Kernel PCA. Center for Robotics and Embedded Systems", University of Southern California, Tech. Rep. CRES-03-010, 2004

6. F. Liu, "Research on The Feature Extraction and Classification based on The Local Structure of The Image in Face Recognition", Nanjing University of Science and Technology, 2015

7. S. Liu, "Subspace Feature Extraction Method and Its Application in Face Recognition", Dalian University of Technology, 2008

8. T. Song, "Research on Facial Image Analysis and Recognition Methods based on Local Features", Zhejiang University, 2015

9. Y. Wang, "Research on Face Recognition Technology based on Image", Jilin University, 2012

10. C.L. Zhang, "Research on Video Tracking Method based on Multi Region Joint Decision Making", Shanghai Jiao Tong University, 2014

11. X. Zhong, "Face Feature Extraction and Recognition based on Ensemble Learning”, Yangzhou University, 2014

Yanhua Wang received her M.S degree from Harbin Normal University. She is a lecturer at Northeast Forestry University. Her research interests include cloud computing and software engineering.

Yaqiu Liu received his PHD degree from Harbin Institute of Technology. He is a professor at Northeast Forestry University. His research interests include cloud computing and software engineering. 